\title{
FOURIER EXPANSIONS AND INTEGRAL REPRESENTATIONS FOR THE APOSTOL-BERNOULLI AND APOSTOL-EULER POLYNOMIALS
}

\author{
QIU-MING LUO
}

\begin{abstract}
We investigate Fourier expansions for the Apostol-Bernoulli and Apostol-Euler polynomials using the Lipschitz summation formula and obtain their integral representations. We give some explicit formulas at rational arguments for these polynomials in terms of the Hurwitz zeta function. We also derive the integral representations for the classical Bernoulli and Euler polynomials and related known results.
\end{abstract}

\section{INTRODUCTION}

The classical Bernoulli polynomials and Euler polynomials are defined by means of the following generating functions (see [1, pp. 804-806] or [18, pp. 25-32])

$$
\frac{z e^{x z}}{e^{z}-1}=\sum_{n=0}^{\infty} B_{n}(x) \frac{z^{n}}{n !} \quad(|z|<2 \pi)
$$

and

$$
\frac{2 e^{x z}}{e^{z}+1}=\sum_{n=0}^{\infty} E_{n}(x) \frac{z^{n}}{n !} \quad(|z|<\pi),
$$

respectively. Obviously, $B_{n}:=B_{n}(0), E_{n}:=2^{n} E_{n}\left(\frac{1}{2}\right)$ are the Bernoulli numbers and Euler numbers respectively.

Some interesting analogues of the classical Bernoulli polynomials and numbers were first investigated by Apostol [2, p. 165, Eq. (3.1)] and (more recently) by Srivastava [20, pp. 83-84]. We begin by recalling here Apostol's definitions as follows:

Received by the editor June 3, 2008 and, in revised form, September 26, 2008.

2000 Mathematics Subject Classification. Primary 11B68; Secondary 42A16, 11M35.

Key words and phrases. Lipschitz summation formula, Fourier expansion, integral representation, Apostol-Bernoulli and Apostol-Euler polynomials and numbers, Bernoulli and Euler polynomials and numbers, Hurwitz Zeta function, Lerch's functional equation, rational arguments.

The author expresses his sincere gratitude to the referee for valuable suggestions and comments. The author thanks Professor Chi-Wang Shu who helped with the submission of this manuscript to the Web submission system of the AMS..

The present investigation was supported in part by the PCSIRT Project of the Ministry of Education of China under Grant \#IRT0621, Innovation Program of Shanghai Municipal Education Committee of China under Grant \#08ZZ24 and Henan Innovation Project For University Prominent Research Talents of China under Grant \#2007KYCX0021.

(C)2009 American Mathematical Society Reverts to public domain 28 years from publication 
Definition 1.1 (Apostol [2]; see also Srivastava 20]). The Apostol-Bernoulli polynomials $\mathcal{B}_{n}(x ; \lambda)$ in $x$ are defined by means of the generating function

$$
\begin{gathered}
\frac{z e^{x z}}{\lambda e^{z}-1}=\sum_{n=0}^{\infty} \mathcal{B}_{n}(x ; \lambda) \frac{z^{n}}{n !} \\
(|z|<2 \pi \text { when } \lambda=1 ;|z|<|\log \lambda| \text { when } \lambda \neq 1)
\end{gathered}
$$

with, of course,

$$
B_{n}(x)=\mathcal{B}_{n}(x ; 1) \quad \text { and } \quad \mathcal{B}_{n}(\lambda):=\mathcal{B}_{n}(0 ; \lambda),
$$

where $\mathcal{B}_{n}(\lambda)$ denotes the so-called Apostol-Bernoulli numbers (in fact, it is a function in $\lambda)$.

Recently, Luo and Srivastava introduced the Apostol-Euler polynomials as follows:

Definition 1.2 (Luo [14]; see also Luo and Srivastava [13]). The Apostol-Euler polynomials $\mathcal{E}_{n}(x ; \lambda)$ in $x$ are defined by means of the generating function

$$
\frac{2 e^{x z}}{\lambda e^{z}+1}=\sum_{n=0}^{\infty} \mathcal{E}_{n}(x ; \lambda) \frac{z^{n}}{n !} \quad(|z|<|\log (-\lambda)|),
$$

with, of course,

$$
E_{n}(x)=\mathcal{E}_{n}(x ; 1) \quad \text { and } \quad \mathcal{E}_{n}(\lambda):=2^{n} \mathcal{E}_{n}\left(\frac{1}{2} ; \lambda\right),
$$

where $\mathcal{E}_{n}(\lambda)$ denote the so-called Apostol-Euler numbers (in fact, it is a function in $\lambda$ ).

Remark 1.3. In Definition 1.1 and Definition [1.2, the original constraints $|z+\log \lambda|<2 \pi$ and $|z+\log \lambda|<\pi$, respectively, should be replaced by the conditions $|z|<2 \pi$ when $\lambda=1 ;|z|<|\log \lambda|$ when $\lambda \neq 1$ and $|z|<|\log (-\lambda)|$ for the referee's clear and detailed argumentation. Hence, the corresponding constraints in References [13], 14], 15] and [20] should also be such.

The Apostol-Bernoulli and Apostol-Euler polynomials have been investigated by many people (see, e.g., 22, 44, [5, [9, [13]-17, [20] and 22]).

D. H. Lehmer [11] gave a new approach to Bernoulli polynomials, starting from a function equation (Rabbe's multiplication theorem). H. Haruki and T. M. Rassias 10. provided the new integral representations for the Bernoulli and Euler polynomials as well as using a similar function equation. Recently, D. Cvijović [7] reproduced the results of H. Haruki and T. M. Rassias in a different way and showed several different integral representations for the Bernoulli and Euler polynomials.

In the present paper, we first investigate Fourier expansions for the ApostolBernoulli and Apostol-Euler polynomials based on the Lipschitz summation formula, and then provide their integral representations. We obtain some explicit formulas for the Apostol-Bernoulli and Apostol-Euler polynomials at rational arguments in terms of the Hurwitz zeta function. We also deduce the corresponding uniform integral representations for the classical Bernoulli and Euler polynomials. We will see that the results of Cvijović or H. Haruki and T. M. Rassias are the corresponding direct consequences of our formulas.

The paper is organized as follows. In the first section we rewrite the definitions of Apostol-Bernoulli and Apostol-Euler polynomials. In the second section we derive 
Fourier expansions for the Apostol-Bernoulli and Apostol-Euler polynomials. In the third section we show their integral representations. In the fourth section we obtain their explicit formulas at rational arguments in terms of the Hurwitz zeta function. In the fifth section we deduce the corresponding uniform integral representations for the classical Bernoulli and Euler polynomials and related results of Cvijović or H. Haruki and T. M. Rassias. In the sixth section we give some applications and remarks; for example, the classical Euler formula $\zeta(2 n)=\frac{(-1)^{n-1}(2 \pi)^{2 n}}{2(2 n) !} B_{2 n}$ is obtained according to our method.

\section{Fourier expansions for the Apostol-Bernoulli And Apostol-Euler polynomials}

In this section we investigate Fourier expansions for the Apostol-Bernoulli and Apostol-Euler polynomials by applying the Lipschitz summation formula.

First we recall the Lipschitz summation formula (see [12] or [19]) as follows:

$$
\sum_{n+\mu>0} \frac{e^{2 \pi i(n+\mu) \tau}}{(n+\mu)^{1-\alpha}}=\frac{\Gamma(\alpha)}{(-2 \pi i)^{\alpha}} \sum_{k \in \mathbb{Z}} \frac{e^{-2 \pi i k \mu}}{(\tau+k)^{\alpha}},
$$

where $\mu \in \mathbb{Z}$ and $\Re(\alpha)>1$ or $\mu \in \mathbb{R} \backslash \mathbb{Z}$ and $\Re(\alpha)>0 ; \tau \in H$ is the complex upper half plane and $\Gamma$ denotes the Gamma function.

Theorem 2.1. For $n=1,0<x<1$ and $n>1,0 \leq x \leq 1, \lambda \in \mathbb{C} \backslash\{0\}$, we have

$$
\begin{aligned}
& \mathcal{B}_{n}(x ; \lambda)=-\delta_{n}(x ; \lambda)-\frac{n !}{\lambda^{x}} \sum^{\prime} \frac{e^{2 \pi i k x}}{(2 \pi i k-\log \lambda)^{n}} \\
&=-\delta_{n}(x ; \lambda)-\frac{n ! i^{n}}{\lambda^{x}}\left[\sum_{k=1}^{\infty} \frac{\exp \left[\left(-2 \pi k x+\frac{n \pi}{2}\right) i\right]}{(2 \pi i k+\log \lambda)^{n}}\right. \\
&\left.+\sum_{k=1}^{\infty} \frac{\exp \left[\left(2 \pi k x-\frac{n \pi}{2}\right) i\right]}{(2 \pi i k-\log \lambda)^{n}}\right],
\end{aligned}
$$

where the symbol $\sum^{\prime}$ denotes the standard convention of a sum over the integers that omits $0 ; \delta_{n}(x ; \lambda)=0$ or $\frac{(-1)^{n} n !}{\lambda^{x} \log ^{n} \lambda}$ according as $\lambda=1$ or $\lambda \neq 1$, respectively.

Proof. For $0 \leq x \leq 1$, by (1.3) and the generalized binomial theorem, we have

$$
\begin{array}{r}
\sum_{k=0}^{\infty} \mathcal{B}_{k}(x ; \lambda) \frac{(2 \pi i \tau)^{k-1}}{k !}=\frac{e^{2 \pi i \tau x}}{\lambda e^{2 \pi i \tau}-1}=-\sum_{k=0}^{\infty} \lambda^{k} e^{2 \pi i(k+x) \tau} \\
\left(|\tau|<1 \text { when } \lambda=1 ;|\tau|<\frac{|\log \lambda|}{2 \pi} \text { when } \lambda \neq 1 ; \Im \tau>\frac{\log |\lambda|}{2 \pi}\right) .
\end{array}
$$

We differentiate both sides of (2.4) with respect to the variable $\tau$, by $n-1$ times and noting that $\mathcal{B}_{0}(x ; \lambda)=\delta_{1, \lambda}$ (see [13, p. 301]). Then we get

$$
\begin{aligned}
& \sum_{k=n}^{\infty} \mathcal{B}_{k}(x ; \lambda) \frac{(2 \pi i)^{k-1} \tau^{k-n}}{k(k-n) !}+\frac{(-1)^{n-1}(n-1) !}{2 \pi i \tau^{n}} \delta_{1, \lambda} \\
&=-(2 \pi i)^{n-1} \sum_{k=0}^{\infty} \lambda^{k}(k+x)^{n-1} e^{2 \pi i(k+x) \tau}
\end{aligned}
$$

where $\delta_{1, \lambda}$ is the Kronecker symbol. 
On the other hand, letting $\alpha=n, \mu \mapsto x, \tau \mapsto \tau+\frac{\log \lambda}{2 \pi i}$ in (2.1), we find that

$$
(-1)^{n}(n-1) ! \sum_{k \in \mathbb{Z}} \frac{e^{-2 \pi i k x}}{[2 \pi i(\tau+k)+\log \lambda]^{n}}=\sum_{k=0}^{\infty} \lambda^{k+x}(k+x)^{n-1} e^{2 \pi i(k+x) \tau} .
$$

Combining (2.5) and (2.6), we obtain

$$
\begin{aligned}
\lambda^{x} \sum_{k=n}^{\infty} \mathcal{B}_{k}(x ; \lambda) \frac{(2 \pi i)^{k-1} \tau^{k-n}}{k(k-n) !}+\lambda^{x} \frac{(-1)^{n-1}(n-1) !}{2 \pi i \tau^{n}} \delta_{1, \lambda} \\
=(-1)^{n-1}(n-1) !(2 \pi i)^{n-1} \sum_{k \in \mathbb{Z}} \frac{e^{-2 \pi i k x}}{[2 \pi i(\tau+k)+\log \lambda]^{n}} .
\end{aligned}
$$

Separating this $k=0$ term in the above sum on the right side yields that

$$
\begin{aligned}
\lambda^{x} \sum_{k=n}^{\infty} & \mathcal{B}_{k}(x ; \lambda) \frac{(2 \pi i)^{k-1} \tau^{k-n}}{k(k-n) !}=(-1)^{n-1}(n-1) !(2 \pi i)^{n-1} \\
& \times \sum^{\prime} \frac{e^{-2 \pi i k x}}{[2 \pi i(\tau+k)+\log \lambda]^{n}}+\frac{(-1)^{n-1}(n-1) !(2 \pi i)^{n-1}}{(2 \pi i \tau+\log \lambda)^{n}}\left(1-\delta_{1, \lambda}\right) .
\end{aligned}
$$

Letting $\tau \rightarrow 0$ in (2.7) we are led at once to the assertion (2.2) of Theorem 2.1.

Noting that $i^{n}=e^{\frac{n \pi i}{2}},(-1)^{n}=e^{-n \pi i}$ and via a simple calculation, then the assertion (2.3) of Theorem 2.1 is a direct consequence of (2.2). This completes our proof.

In the same manner, we may prove the following.

Theorem 2.2. For $n=0,0<x<1$ and $n>0,0 \leq x \leq 1, \lambda \in \mathbb{C} \backslash\{0,-1\}$, we have

$$
\begin{aligned}
& \mathcal{E}_{n}(x ; \lambda)=\frac{2 \cdot n !}{\lambda^{x}} \sum_{k \in \mathbb{Z}} \frac{e^{(2 k-1) \pi i x}}{[(2 k-1) \pi i-\log \lambda]^{n+1}} \\
&=\frac{2 \cdot n ! i^{n+1}}{\lambda^{x}}\left[\sum_{k=0}^{\infty} \frac{\exp \left[\left(\frac{n+1}{2} \pi-(2 k+1) \pi x\right) i\right]}{[(2 k+1) \pi i+\log \lambda]^{n+1}}\right. \\
& \\
&\left.\quad+\sum_{k=0}^{\infty} \frac{\exp \left[\left(-\frac{n+1}{2} \pi+(2 k+1) \pi x\right) i\right]}{[(2 k+1) \pi i-\log \lambda]^{n+1}}\right] .
\end{aligned}
$$

By Theorem 2.1 and Theorem 2.2, we can deduce respectively the Fourier expansions for the classical Bernoulli and Euler polynomials as follows:

Corollary 2.3. For $n=1,0<x<1$ and $n>1,0 \leq x \leq 1$, we have

$$
\begin{aligned}
B_{n}(x) & =-\frac{n !}{(2 \pi i)^{n}} \sum^{\prime} \frac{e^{2 \pi i k x}}{k^{n}} \\
& =-\frac{2 \cdot n !}{(2 \pi)^{n}} \sum_{k=1}^{\infty} \frac{\cos \left(2 \pi k x-\frac{n \pi}{2}\right)}{k^{n}} .
\end{aligned}
$$


Corollary 2.4. For $n=0,0<x<1$ and $n>0,0 \leq x \leq 1$, we have

$$
\begin{aligned}
E_{n}(x) & =\frac{2 \cdot n !}{(\pi i)^{n+1}} \sum_{k \in \mathbb{Z}} \frac{e^{(2 k-1) \pi i x}}{(2 k-1)^{n+1}} \\
& =\frac{4 \cdot n !}{\pi^{n+1}} \sum_{k=0}^{\infty} \frac{\sin \left[(2 k+1) \pi x-\frac{n \pi}{2}\right]}{(2 k+1)^{n+1}} .
\end{aligned}
$$

Remark 2.5. Replacing $\tau$ by $\tau+\frac{\log \lambda}{2 \pi i}+\frac{1}{2}$ in (2.1) and applying $\mathcal{E}_{0}(x ; \lambda)=\frac{2}{\lambda+1}$ (see, for details, [13]-15]) when we prove the assertion (2.8) of Theorem 2.2.

Remark 2.6. We define the $n$-th Apostol-Bernoulli function as

$$
\widehat{\mathcal{B}}_{n}(x ; \lambda):=\mathcal{B}_{n}(x ; \lambda)(0 \leq x<1), \quad \widehat{\mathcal{B}}_{n}(x+1 ; \lambda)=\lambda^{-1} \widehat{\mathcal{B}}_{n}(x ; \lambda),
$$

which is also called the quasi-periodicity Apostol-Bernoulli polynomials. For any $x \in \mathbb{R}, r \in \mathbb{Z}$, we have

$$
\widehat{\mathcal{B}}_{n}(x ; \lambda)=\lambda^{-[x]} \mathcal{B}_{n}(\{x\} ; \lambda), \quad \widehat{\mathcal{B}}_{n}(x+r ; \lambda)=\lambda^{-r} \widehat{\mathcal{B}}_{n}(x ; \lambda) .
$$

Here the notation $\{x\}$ denotes the fractional part of $x$, and the notation $[x]$ denotes the greatest integer not exceeding $x$.

Clearly, the Apostol-Bernoulli polynomials $\mathcal{B}_{n}(x ; \lambda)(0 \leq x<1)$ are the quasiperiodicity functions in $x$ with period 1 . One of the special cases of the quasiperiodicity Apostol-Bernoulli polynomials is just Carlitz's periodic Bernoulli function [3, p. 661] for $\lambda=1$.

Remark 2.7. We define the $n$-th Apostol-Euler function as

$$
\widehat{\mathcal{E}}_{n}(x ; \lambda):=\mathcal{E}_{n}(x ; \lambda)(0 \leq x<1), \quad \widehat{\mathcal{E}}_{n}(x+1 ; \lambda)=-\lambda^{-1} \widehat{\mathcal{E}}_{n}(x ; \lambda),
$$

which is called the quasi-periodicity Apostol-Euler polynomials. For any $x \in \mathbb{R}, r \in$ $\mathbb{Z}$, we have

$$
\widehat{\mathcal{E}}_{n}(x ; \lambda)=(-1)^{[x]} \lambda^{-[x]} \mathcal{E}_{n}(\{x\} ; \lambda), \quad \widehat{\mathcal{E}}_{n}(x+r ; \lambda)=(-1)^{r} \lambda^{-r} \widehat{\mathcal{E}}_{n}(x ; \lambda) .
$$

Obviously, the Apostol-Euler polynomials $\mathcal{E}_{n}(x ; \lambda)(0 \leq x<1)$ are the quasiperiodicity functions in $x$ with period 1 . One of the special cases of the quasiperiodicity Apostol-Euler polynomials is just Carlitz's periodic Euler function [3. p. 661] for $\lambda=1$. 
Remark 2.8. We employ a useful relationship [15, p. 636, Eq. (38)]

$$
\mathcal{E}_{n}(x ; \lambda)=\frac{2}{n+1}\left[\mathcal{B}_{n+1}(x ; \lambda)-2^{n+1} \mathcal{B}_{n+1}\left(\frac{x}{2} ; \lambda^{2}\right)\right]
$$

to (2.2) and (2.3), respectively; we can also arrive at the corresponding (2.8) and (2.9).

Remark 2.9. Throughout this paper, we take the principal value of the logarithm $\log \lambda$, i.e., $\log \lambda=\log |\lambda|+i \arg \lambda(-\pi<\arg \lambda \leq \pi)$ when $\lambda \neq 1$; We choose $\log 1=0$ when $\lambda=1$.

\section{Integral Representations for the Apostol-Bernoulli And Apostol-Euler polynomials}

In this section we give the integral representations for the Apostol-Bernoulli and Apostol-Euler polynomials with their Fourier expansions. For convenience, we take $\lambda=e^{2 \pi i \xi}(\xi \in \mathbb{R},|\xi|<1)$ in this section.

Theorem 3.1. For $n=1,2, \ldots, 0 \leq \Re(x) \leq 1,|\xi|<1, \xi \in \mathbb{R}$, we have

$$
\begin{aligned}
& \mathcal{B}_{n}\left(x ; e^{2 \pi i \xi}\right)=-\Delta_{n}(x ; \xi) \\
& \quad-n e^{-2 \pi i x \xi} \int_{0}^{\infty} \frac{U(n ; x, t) \cosh (2 \pi \xi t)+i V(n ; x, t) \sinh (2 \pi \xi t)}{\cosh 2 \pi t-\cos 2 \pi x} t^{n-1} \mathrm{~d} t
\end{aligned}
$$

where $\Delta_{n}(x ; \xi)=0$ or $\frac{(-1)^{n} n !}{e^{2 \pi i x \xi}(2 \pi i \xi)^{n}}$ according as $\xi=0$ or $\xi \neq 0$, respectively, and

$$
\begin{aligned}
& U(n ; x, t)=\left[\cos \left(2 \pi x-\frac{n \pi}{2}\right)-\cos \left(\frac{n \pi}{2}\right) e^{-2 \pi t}\right] \\
& V(n ; x, t)=\left[\sin \left(2 \pi x-\frac{n \pi}{2}\right)+\sin \left(\frac{n \pi}{2}\right) e^{-2 \pi t}\right]
\end{aligned}
$$

Proof. Returning to (2.2) and setting $\lambda=e^{2 \pi i \xi}, k \mapsto-k$ yields

$$
\mathcal{B}_{n}\left(x ; e^{2 \pi i \xi}\right)=-\Delta_{n}(x ; \xi)-\frac{n ! e^{-2 \pi i x \xi}}{(-2 \pi i)^{n}} \sum^{\prime} \frac{e^{-2 \pi i k x}}{(k+\xi)^{n}} .
$$

Using the known integral formula

$$
\int_{0}^{\infty} t^{n} e^{-a t} \mathrm{~d} t=\frac{n !}{a^{n+1}} \quad(n=0,1, \ldots ; \Re(a)>0),
$$

and noting that $\left(-\frac{1}{i}\right)^{n}=e^{\frac{n \pi i}{2}}$ and $(-1)^{n}=e^{-n \pi i}$, then we have 


$$
\begin{aligned}
& \mathcal{B}_{n}\left(x ; e^{2 \pi i \xi}\right)=-\Delta_{n}(x ; \xi)-\frac{n e^{-2 \pi i x \xi}}{(-2 \pi i)^{n}}\left\{\sum_{k=1}^{\infty} e^{-2 \pi i k x} \int_{0}^{\infty} t^{n-1} e^{-(k+\xi) t} \mathrm{~d} t\right. \\
& \left.+(-1)^{n} \sum_{k=1}^{\infty} e^{2 \pi i k x} \int_{0}^{\infty} t^{n-1} e^{-(k-\xi) t} \mathrm{~d} t\right\} \\
& =-\Delta_{n}(x ; \xi)-\frac{n e^{-2 \pi i x \xi}}{(-2 \pi i)^{n}}\left\{\int_{0}^{\infty} e^{-\xi t} t^{n-1} \sum_{k=1}^{\infty} e^{-(2 \pi i x+t) k} \mathrm{~d} t\right. \\
& \left.+(-1)^{n} \int_{0}^{\infty} e^{\xi t} t^{n-1} \sum_{k=1}^{\infty} e^{(2 \pi i x-t) k} \mathrm{~d} t\right\} \\
& =-\Delta_{n}(x ; \xi)-\frac{n e^{-2 \pi i x \xi}}{(-2 \pi i)^{n}}\left\{\int_{0}^{\infty} \frac{e^{-2 \pi i x}}{e^{t}-e^{-2 \pi i x}} e^{-\xi t} t^{n-1} \mathrm{~d} t\right. \\
& \left.+(-1)^{n} \int_{0}^{\infty} \frac{e^{2 \pi i x}}{e^{t}-e^{2 \pi i x}} e^{\xi t} t^{n-1} \mathrm{~d} t\right\} \\
& =-\Delta_{n}(x ; \xi)-\frac{n e^{-2 \pi i x \xi}}{2(2 \pi)^{n}}\left\{\int_{0}^{\infty} \frac{e^{\frac{n \pi i}{2}}\left(e^{-2 \pi i x}-e^{-t}\right)}{\cosh t-\cos 2 \pi x} e^{-\xi t} t^{n-1} \mathrm{~d} t\right. \\
& \left.+\int_{0}^{\infty} \frac{e^{-\frac{n \pi i}{2}}\left(e^{2 \pi i x}-e^{-t}\right)}{\cosh t-\cos 2 \pi x} e^{\xi t} t^{n-1} \mathrm{~d} t\right\}
\end{aligned}
$$

It follows that we make the transformation $t=2 \pi u$, and after simplification we obtain the desired (3.1) immediately. This completes the proof.

We can obtain the following integral representations for the Apostol-Euler polynomials by a similar method.

Theorem 3.2. For $n=1,2, \ldots, 0 \leq \Re(x) \leq 1,|\xi|<\frac{1}{2}, \xi \in \mathbb{R}$, we have

$$
\begin{aligned}
\mathcal{E}_{n}\left(x ; e^{2 \pi i \xi}\right)=2 e^{-2 \pi i x \xi} & \\
& \times \int_{0}^{\infty} \frac{X(n ; x, t) \cosh (2 \pi \xi t)+i Y(n ; x, t) \sinh (2 \pi \xi t)}{\cosh 2 \pi t-\cos 2 \pi x} t^{n} \mathrm{~d} t,
\end{aligned}
$$

where

$$
\begin{aligned}
& X(n ; x, t)=\left[e^{-\pi t} \sin \left(\pi x+\frac{n \pi}{2}\right)+e^{\pi t} \sin \left(\pi x-\frac{n \pi}{2}\right)\right] \\
& Y(n ; x, t)=\left[e^{-\pi t} \cos \left(\pi x+\frac{n \pi}{2}\right)-e^{\pi t} \cos \left(\pi x-\frac{n \pi}{2}\right)\right] .
\end{aligned}
$$

On the other hand, we can also arrive at the following different integral representations for the Apostol-Bernoulli and Apostol-Euler polynomials.

Theorem 3.3. For $n=1,2, \ldots, 0 \leq \Re(x) \leq 1,|\xi|<1, \xi \in \mathbb{R}$, we have

$$
\begin{aligned}
& \mathcal{B}_{n}\left(x ; e^{2 \pi i \xi}\right)=-\Delta_{n}(x ; \xi)+\frac{2 n e^{-2 \pi i x \xi}}{(-2 \pi)^{n}} \\
& \quad \times \int_{0}^{1} \frac{U^{\prime}(n ; x, t) \cosh (\xi \log t)-i V^{\prime}(n ; x, t) \sinh (\xi \log t)}{t^{2}-2 t \cos 2 \pi x+1}(\log t)^{n-1} \mathrm{~d} t
\end{aligned}
$$


where $\Delta_{n}(x ; \xi)=0$ or $\frac{(-1)^{n} n !}{e^{2 \pi i x \xi}(2 \pi i \xi)^{n}}$ according as $\xi=0$ or $\xi \neq 0$, respectively, and

$$
\begin{aligned}
U^{\prime}(n ; x, t) & =\left[\cos \left(2 \pi x-\frac{n \pi}{2}\right)-t \cos \left(\frac{n \pi}{2}\right)\right], \\
V^{\prime}(n ; x, t) & =\left[\sin \left(2 \pi x-\frac{n \pi}{2}\right)+t \sin \left(\frac{n \pi}{2}\right)\right] .
\end{aligned}
$$

Proof. First we substitute $\cosh 2 \pi t=\frac{e^{2 \pi t}+e^{-2 \pi t}}{2}$ into (3.1). Then we see that

$$
\begin{aligned}
\mathcal{B}_{n}\left(x ; e^{2 \pi i \xi}\right)= & -\Delta_{n}(x ; \xi)-2 n e^{-2 \pi i x \xi} \\
& \times \int_{0}^{\infty} \frac{U(n ; x, t) \cosh (2 \pi \xi t)+i V(n ; x, t) \sinh (2 \pi \xi t)}{e^{2 \pi t}+e^{-2 \pi t}-2 \cos 2 \pi x} t^{n-1} \mathrm{~d} t .
\end{aligned}
$$

Then making the transformation $u=e^{-2 \pi t}$ in (3.5), we easily obtain formula (3.4). This completes the proof.

Similarly, we obtain

Theorem 3.4. For $n=1,2, \ldots, 0 \leq \Re(x) \leq 1,|\xi|<\frac{1}{2}, \xi \in \mathbb{R}$, we have

$$
\begin{aligned}
\mathcal{E}_{n}\left(x ; e^{2 \pi i \xi}\right) & =(-1)^{n} \frac{4 e^{-2 \pi i x \xi}}{\pi^{n+1}} \\
& \times \int_{0}^{1} \frac{X^{\prime}(n ; x, t) \cosh (2 \xi \log t)-i Y^{\prime}(n ; x, t) \sinh (2 \xi \log t)}{t^{4}-2 t^{2} \cos 2 \pi x+1}(\log t)^{n} \mathrm{~d} t,
\end{aligned}
$$

where

$$
\begin{aligned}
& X^{\prime}(n ; x, t)=\left[t^{2} \sin \left(\pi x+\frac{n \pi}{2}\right)+\sin \left(\pi x-\frac{n \pi}{2}\right)\right], \\
& Y^{\prime}(n ; x, t)=\left[t^{2} \cos \left(\pi x+\frac{n \pi}{2}\right)-\cos \left(\pi x-\frac{n \pi}{2}\right)\right] .
\end{aligned}
$$

Remark 3.5. For any integers $\ell$, we see easily that $\mathcal{B}_{n}\left(x ; e^{2 \pi i(\ell+\xi)}\right)=\mathcal{B}_{n}\left(x ; e^{2 \pi i \xi}\right)$, $\mathcal{E}_{n}\left(x ; e^{2 \pi i(\ell+\xi)}\right)=\mathcal{E}_{n}\left(x ; e^{2 \pi i \xi}\right)$. Therefore, the Apostol-Bernoulli polynomials $\mathcal{B}_{n}\left(x ; e^{2 \pi i \xi}\right)$ and the Apostol-Euler polynomials $\mathcal{E}_{n}\left(x ; e^{2 \pi i \xi}\right)$ are the periodicity functions in $\xi$ with period $2 \pi$. In view of this observation we say that $\xi$ may take any real numbers in Theorem 3.1.Theorem 3.4.

Remark 3.6. We can also prove Theorem 2.1 and Theorem 2.2 by Theorem 3.1 and Theorem 3.2 , respectively, in an inverse process.

\section{Explicit formulas for the Apostol-Bernoulli and Apostol-Euler POLYNOMIALS AT RATIONAL ARGUMENTS}

In this section we obtain some explicit formulas for the Apostol-Bernoulli and Apostol-Euler polynomials at rational arguments. We can see that some known formulas of Cvijović and Klinowski are the corresponding special cases of our formulas.

The Hurwitz-Lerch zeta function $\Phi(z, s, a)$ defined by (cf., e.g., [21, p. 121, et seq.])

$$
\begin{gathered}
\Phi(z, s, a):=\sum_{n=0}^{\infty} \frac{z^{n}}{(n+a)^{s}} \\
\left(a \in \mathbb{C} \backslash \mathbb{Z}_{0}^{-} ; s \in \mathbb{C} \text { when }|z|<1 ; \mathfrak{R}(s)>1 \text { when }|z|=1\right)
\end{gathered}
$$


contains, as its special cases, not only the Riemann and Hurwitz zeta functions

$$
\zeta(s):=\Phi(1, s, 1)=\zeta(s, 1)=\frac{1}{2^{s}-1} \zeta\left(s, \frac{1}{2}\right)=\sum_{n=1}^{\infty} \frac{1}{n^{s}}
$$

and

$$
\zeta(s, a):=\Phi(1, s, a)=\sum_{n=0}^{\infty} \frac{1}{(n+a)^{s}} \quad\left(\Re(s)>1 ; a \notin \mathbb{Z}_{0}^{-}\right)
$$

and the Lerch zeta function (or periodic zeta function)

$$
\begin{gathered}
l_{s}(\xi):=\sum_{n=1}^{\infty} \frac{e^{2 n \pi i \xi}}{n^{s}}=e^{2 \pi i \xi} \Phi\left(e^{2 \pi i \xi}, s, 1\right) \\
(\xi \in \mathbb{R} ; \Re(s)>1),
\end{gathered}
$$

but also such other functions as the polylogarithmic function

$$
\begin{gathered}
\operatorname{Li}_{s}(z):=\sum_{n=1}^{\infty} \frac{z^{n}}{n^{s}}=z \Phi(z, s, 1) \\
(s \in \mathbb{C} \quad \text { when } \quad|z|<1 ; \mathfrak{R}(s)>1 \quad \text { when } \quad|z|=1)
\end{gathered}
$$

and the Lipschitz-Lerch zeta function (cf. [21, p. 122, Eq. 2.5 (11)])

$$
\begin{gathered}
\phi(\xi, a, s):=\sum_{n=0}^{\infty} \frac{e^{2 n \pi i \xi}}{(n+a)^{s}}=\Phi\left(e^{2 \pi i \xi}, s, a\right)=: L(\xi, s, a) \\
\left(a \in \mathbb{C} \backslash \mathbb{Z}_{0}^{-} ; \mathfrak{R}(s)>0 \text { when } \xi \in \mathbb{R} \backslash \mathbb{Z} ; \mathfrak{R}(s)>1 \text { when } \xi \in \mathbb{Z}\right),
\end{gathered}
$$

which was first studied by Rudolf Lipschitz (1832-1903) and Matyáš Lerch (18601922) in connection with Dirichlet's famous theorem on primes in arithmetic progressions.

Recently, H. M. Srivastava made use of Apostol's formula (see [2, p. 164])

$$
\phi(\xi, a, 1-n)=\Phi\left(e^{2 \pi i \xi}, 1-n, a\right)=-\frac{\mathcal{B}_{n}\left(a ; e^{2 \pi i \xi}\right)}{n} \quad(n \in \mathbb{N})
$$

and Lerch's functional equation (see [2, p. 161, (1.4)])

$$
\begin{aligned}
& \phi(\xi, a, 1-s)=\frac{\Gamma(s)}{(2 \pi)^{s}}\{\exp [\left.\left(\frac{1}{2} s-2 a \xi\right) \pi i\right] \phi(-a, \xi, s) \\
&+\left.\exp \left[\left(-\frac{1}{2} s+2 a(1-\xi)\right) \pi i\right] \phi(a, 1-\xi, s)\right\} \\
&(s \in \mathbb{C} ; 0<\xi<1)
\end{aligned}
$$


to derive the following formula of Apostol-Bernoulli polynomials at rational arguments (see [20, p. 84, Eq. (4.6)]):

$$
\begin{aligned}
& \mathcal{B}_{n}\left(\frac{p}{q} ; e^{2 \pi i \xi}\right)=-\frac{n !}{(2 q \pi)^{n}}\left\{\sum_{j=1}^{q} \zeta\left(n, \frac{\xi+j-1}{q}\right) \exp \left[\left(\frac{n}{2}-\frac{2(\xi+j-1) p}{q}\right) \pi i\right]\right. \\
& \left.+\sum_{j=1}^{q} \zeta\left(n, \frac{j-\xi}{q}\right) \exp \left[\left(-\frac{n}{2}+\frac{2(j-\xi) p}{q}\right) \pi i\right]\right\} \\
& (n \in \mathbb{N} \backslash\{1\} ; q \in \mathbb{N} ; p \in \mathbb{Z} ; \xi \in \mathbb{R}) \text {. }
\end{aligned}
$$

Below we obtain similar formulas by using Fourier expansions for the ApostolBernoulli polynomials and Apostol-Euler polynomials, respectively.

Theorem 4.1. For $n \in \mathbb{N} \backslash\{1\}, q \in \mathbb{N}, p \in \mathbb{Z}, \xi \in \mathbb{R}$, $|\xi|<1$, the following formula of Apostol-Bernoulli polynomials at rational arguments

$$
\begin{aligned}
\mathcal{B}_{n}\left(\frac{p}{q} ; e^{2 \pi i \xi}\right)=-\Delta_{n} & \left(\frac{p}{q} ; \xi\right)-\frac{n !}{(2 \pi q)^{n}}\left\{\sum_{j=1}^{q} \zeta\left(n, \frac{j+\xi}{q}\right) \exp \left[\left(\frac{n}{2}-\frac{2(j+\xi) p}{q}\right) \pi i\right]\right. \\
& \left.+\sum_{j=1}^{q} \zeta\left(n, \frac{j-\xi}{q}\right) \exp \left[\left(-\frac{n}{2}+\frac{2(j-\xi) p}{q}\right) \pi i\right]\right\}
\end{aligned}
$$

holds true in terms of the Hurwitz zeta function, where $\Delta_{n}(x ; \xi)=0$ or $\frac{(-1)^{n} n !}{e^{2 \pi i x \xi}(2 \pi i \xi)^{n}}$ according as $\xi=0$ or $\xi \neq 0$, respectively.

Proof. We employ formula (2.3),

$$
\mathcal{B}_{n}(x ; \lambda)=-\delta_{n}(x ; \lambda)-\frac{n ! i^{n}}{\lambda^{x}}\left[\sum_{k=1}^{\infty} \frac{\exp \left[\left(-2 \pi k x+\frac{n \pi}{2}\right) i\right]}{(2 \pi i k+\log \lambda)^{n}}+\sum_{k=1}^{\infty} \frac{\exp \left[\left(2 \pi k x-\frac{n \pi}{2}\right) i\right]}{(2 \pi i k-\log \lambda)^{n}}\right],
$$

so that, in view of the definition (4.1) and the elementary series identity

$$
\sum_{k=1}^{\infty} f(k)=\sum_{j=1}^{\ell} \sum_{k=0}^{\infty} f(\ell k+j) \quad(\ell \in \mathbb{N})
$$

we find the formula:

$$
\begin{aligned}
\mathcal{B}_{n}(x ; \lambda)= & -\delta_{n}(x ; \lambda)-\frac{n ! i^{n} \lambda^{-x}}{(2 \pi i \ell)^{n}} \\
\times & {\left[\sum_{j=1}^{\ell} \Phi\left(e^{2 \pi i \ell x}, n, \frac{2 \pi i j-\log \lambda}{2 \pi i \ell}\right) \exp \left[\left(2 \pi j x-\frac{n \pi}{2}\right) i\right]\right.} \\
& \left.+\sum_{j=1}^{\ell} \Phi\left(e^{-2 \pi i \ell x}, n, \frac{2 \pi i j+\log \lambda}{2 \pi i \ell}\right) \exp \left[-\left(2 \pi j x+\frac{n \pi}{2}\right) i\right]\right] .
\end{aligned}
$$

Setting $\lambda=\exp (2 \pi i \xi), x=\frac{p}{q}, \ell=q$ in (4.13), we then obtain the assertion of Theorem 4.1. This completes the proof. 
If we make use of the equivalent of (2.9) as

$$
\begin{aligned}
\mathcal{E}_{n}(x ; \lambda)=\frac{2 \cdot n ! i^{n+1}}{\lambda^{x}} & {\left[\sum_{k=1}^{\infty} \frac{\exp \left[\left(\frac{n+1}{2} \pi-(2 k-1) \pi x\right) i\right]}{[(2 k-1) \pi i+\log \lambda]^{n+1}}\right.} \\
& \left.+\sum_{k=1}^{\infty} \frac{\exp \left[\left(-\frac{n+1}{2} \pi+(2 k-1) \pi x\right) i\right]}{[(2 k-1) \pi i-\log \lambda]^{n+1}}\right]
\end{aligned}
$$

and the elementary series identity (4.12), by an analogous method, we provide that

Theorem 4.2. For $n, q \in \mathbb{N}, p \in \mathbb{Z}, \xi \in \mathbb{R},|\xi|<1$, the following formula of Apostol-Euler polynomials at rational arguments

$$
\begin{aligned}
\mathcal{E}_{n}\left(\frac{p}{q} ; e^{2 \pi i \xi}\right)=\frac{2 \cdot n !}{(2 q \pi)^{n+1}} \\
\quad \times\left\{\sum_{j=1}^{q} \zeta\left(n+1, \frac{2 j+2 \xi-1}{2 q}\right) \exp \left[\left(\frac{n+1}{2}-\frac{(2 j+2 \xi-1) p}{q}\right) \pi i\right]\right. \\
\left.\quad+\sum_{j=1}^{q} \zeta\left(n+1, \frac{2 j-2 \xi-1}{2 q}\right) \exp \left[\left(-\frac{n+1}{2}+\frac{(2 j-2 \xi-1) p}{q}\right) \pi i\right]\right\}
\end{aligned}
$$

holds true in terms of the Hurwitz zeta function.

Upon the special cases of (4.11) and (4.15), for $\xi=0$, are respectively the following known results given earlier by Cvijović and Klinowski.

Corollary 4.3 ([6, p. 1529, Theorem A]). For $n \in \mathbb{N} \backslash\{1\}, q \in \mathbb{N}, p \in \mathbb{Z}$, the following formula for the classical Bernoulli polynomials

$$
B_{n}\left(\frac{p}{q}\right)=-\frac{2 \cdot n !}{(2 q \pi)^{n}} \sum_{j=1}^{q} \zeta\left(n, \frac{j}{q}\right) \cos \left(\frac{2 j p \pi}{q}-\frac{n \pi}{2}\right)
$$

holds true.

Corollary 4.4 ([6, p. 1529, Theorem B]). For $n, q \in \mathbb{N}, p \in \mathbb{Z}$, the following formula for the classical Euler polynomials

$$
E_{n}\left(\frac{p}{q}\right)=\frac{4 \cdot n !}{(2 q \pi)^{n+1}} \sum_{j=1}^{q} \zeta\left(n+1, \frac{2 j-1}{2 q}\right) \sin \left(\frac{(2 j-1) p \pi}{q}-\frac{n \pi}{2}\right)
$$

holds true.

Remark 4.5. We may also prove formula (4.15) by applying the relationship (2.18) and Theorem 4.1 .

Remark 4.6. In view of Remark 3.5, we say that $\xi$ is any real number in Theorem 4.1 and Theorem 4.2 .

Remark 4.7. Obviously, Srivastava's formula (4.9) is an equivalent with our formula (4.11). 


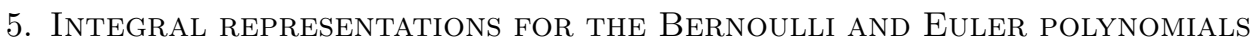

In this section we will see that Theorem 5.1 and Theorem 5.2 below involve the results of Cvijović or H. Haruki and T. M. Rassias.

By (3.1) and (3.3) for $\xi=0$, it follows that we give the uniform integral representations for the classical Bernoulli and Euler polynomials, respectively.

Theorem 5.1. For $n=1,2, \ldots, 0 \leq \Re(x) \leq 1$, we have

$$
B_{n}(x)=-n \int_{0}^{\infty} \frac{\cos \left(2 \pi x-\frac{n \pi}{2}\right)-e^{-2 \pi t} \cos \left(\frac{n \pi}{2}\right)}{\cosh 2 \pi t-\cos 2 \pi x} t^{n-1} \mathrm{~d} t .
$$

Theorem 5.2. For $n=1,2, \ldots, 0 \leq \Re(x) \leq 1$, we have

$$
E_{n}(x)=2 \int_{0}^{\infty} \frac{e^{\pi t} \sin \left(\pi x-\frac{n \pi}{2}\right)+e^{-\pi t} \sin \left(\pi x+\frac{n \pi}{2}\right)}{\cosh 2 \pi t-\cos 2 \pi x} t^{n} \mathrm{~d} t .
$$

Remark 5.3. Theorem 5.1 and Theorem 5.2 above show the uniform integral representations for the classical Bernoulli and Euler polynomials which were never found in the classical literature, for example [1], 8] and [18, etc. So these uniform formulas are interesting in this subject.

By (3.4) and (3.6) for $\xi=0$, we easily find the following additional integral representations for the classical Bernoulli and Euler polynomials, respectively.

Theorem 5.4. For $n=1,2, \ldots, 0 \leq \Re(x) \leq 1$, we have

$$
B_{n}(x)=(-1)^{n} \frac{2 n}{(2 \pi)^{n}} \int_{0}^{1} \frac{\cos \left(2 \pi x-\frac{n \pi}{2}\right)-t \cos \left(\frac{n \pi}{2}\right)}{t^{2}-2 t \cos 2 \pi x+1}(\log t)^{n-1} \mathrm{~d} t .
$$

Theorem 5.5. For $n=1,2, \ldots, 0 \leq \Re(x) \leq 1$, we have

$$
E_{n}(x)=(-1)^{n} \frac{4}{\pi^{n+1}} \int_{0}^{1} \frac{\sin \left(\pi x-\frac{n \pi}{2}\right)+t^{2} \sin \left(\pi x+\frac{n \pi}{2}\right)}{t^{4}-2 t^{2} \cos 2 \pi x+1}(\log t)^{n} \mathrm{~d} t .
$$

We see easily that Theorem 5.4 and Theorem 5.5 imply the main results of Cvijović 7, p. 170, Theorem 1] or H. Haruki and T. M. Rassias [10, p. 82, Theorem (ii)(iv)], i.e.,

$$
\begin{aligned}
& B_{2 n}(x)=(-1)^{n} \frac{2(2 n)}{(2 \pi)^{2 n}} \int_{0}^{1} \frac{\cos 2 \pi x-t}{t^{2}-2 t \cos 2 \pi x+1}(\log t)^{2 n-1} \mathrm{~d} t, \\
& B_{2 n-1}(x)=(-1)^{n} \frac{2(2 n-1)}{(2 \pi)^{2 n-1}} \int_{0}^{1} \frac{\sin 2 \pi x}{t^{2}-2 t \cos 2 \pi x+1}(\log t)^{2 n-2} \mathrm{~d} t, \\
& E_{2 n}(x)=(-1)^{n} \frac{4}{\pi^{2 n+1}} \int_{0}^{1} \frac{\left(t^{2}+1\right) \sin \pi x}{t^{4}-2 t^{2} \cos 2 \pi x+1}(\log t)^{2 n} \mathrm{~d} t, \\
& E_{2 n-1}(x)=(-1)^{n} \frac{4}{\pi^{2 n}} \int_{0}^{1} \frac{\left(t^{2}-1\right) \cos \pi x}{t^{4}-2 t^{2} \cos 2 \pi x+1}(\log t)^{2 n-1} \mathrm{~d} t .
\end{aligned}
$$

On the other hand, Theorem 5.1 and Theorem 5.2 also imply the classical integral representations for the Bernoulli polynomials and Euler polynomials, respectively 
(see [18, pp. 27, 31]):

$$
\begin{aligned}
& B_{2 n}(x)=(-1)^{n+1}(2 n) \int_{0}^{\infty} \frac{\cos 2 \pi x-e^{-2 \pi t}}{\cosh 2 \pi t-\cos 2 \pi x} t^{2 n-1} \mathrm{~d} t, \\
& B_{2 n-1}(x)=(-1)^{n}(2 n-1) \int_{0}^{\infty} \frac{\sin 2 \pi x}{\cosh 2 \pi t-\cos 2 \pi x} t^{2 n-2} \mathrm{~d} t \\
& E_{2 n}(x)=4(-1)^{n} \int_{0}^{\infty} \frac{\sin \pi x \cosh \pi t}{\cosh 2 \pi t-\cos 2 \pi x} t^{2 n} \mathrm{~d} t \\
& E_{2 n-1}(x)=4(-1)^{n} \int_{0}^{\infty} \frac{\cos \pi x \sinh \pi t}{\cosh 2 \pi t-\cos 2 \pi x} t^{2 n-1} \mathrm{~d} t .
\end{aligned}
$$

Remark 5.6. If we make an appropriate transformation $u=e^{-2 \pi t}$ in (5.9) and (5.10), and make a suitable transformation $u=e^{-\pi t}$ in (5.11) and (5.12), respectively, then we can directly obtain (5.5) -(5.8); i.e., the main results of Cvijović or H. Haruki and T. M. Rassias are only a very simple transmogrification for the corresponding classical cases (5.9)-(5.12). Therefore, in view of this reason, we say that (5.5) -(5.8) are not new integral representations for the classical Bernoulli polynomials and Euler polynomials.

\section{Further observations AND CONSEQUences}

By formula (2.3) of Theorem 2.1 for $x=0$, we obtain the relationship between the Apostol-Bernoulli numbers and the Hurwitz zeta function as follows:

$$
\mathcal{B}_{n}(\lambda)=\frac{(-1)^{n-1} n !}{(2 \pi i)^{n}}\left[(-1)^{n} \zeta\left(n, 1-\frac{\log \lambda}{2 \pi i}\right)+\zeta\left(n, \frac{\log \lambda}{2 \pi i}\right)\right] .
$$

Letting $\lambda=1, n \mapsto 2 n$ in (6.1), we at once produce the following famous Euler formula (see, e.g, [1, p. 807, 23.2.16]):

$$
\zeta(2 n)=\frac{(-1)^{n-1}(2 \pi)^{2 n}}{2(2 n) !} B_{2 n} .
$$

On the other hand, we define zeta functions as

$$
\beta(n ; \xi)=\sum_{k=0}^{\infty} \frac{(-1)^{k}}{(2 k+2 \xi+1)^{n}}, \quad \beta(n)=\sum_{k=0}^{\infty} \frac{(-1)^{k}}{(2 k+1)^{n}} \quad(\xi \in \mathbb{R}) .
$$

Setting $\lambda=e^{2 \pi i \xi}$ in (2.9) of Theorem 2.2 we have

$$
\begin{aligned}
\mathcal{E}_{n}\left(x ; e^{2 \pi i \xi}\right)=\frac{2 \cdot n !}{\pi^{n+1} e^{2 \pi i \xi x}}[ & \sum_{k=0}^{\infty} \frac{\exp \left[\left(\frac{n+1}{2} \pi-(2 k+1) \pi x\right) i\right]}{(2 k+2 \xi+1)^{n+1}} \\
& \left.+\sum_{k=0}^{\infty} \frac{\exp \left[\left(-\frac{n+1}{2} \pi+(2 k+1) \pi x\right) i\right]}{(2 k-2 \xi+1)^{n+1}}\right] .
\end{aligned}
$$

Taking $x=\frac{1}{2}$ in (6.4) and noting that $\mathcal{E}_{n}(\lambda)=2^{n} \mathcal{E}_{n}\left(\frac{1}{2} ; \lambda\right)$ and (6.3), we readily obtain the following relationship between the Apostol-Euler numbers $\mathcal{E}_{n}\left(e^{2 \pi i \xi}\right)$ and the zeta function $\beta(n ; \xi)$ :

$$
\mathcal{E}_{n}\left(e^{2 \pi i \xi}\right)=\frac{2^{n+1} i^{n} \cdot n !}{\pi^{n+1} e^{\pi i \xi}}\left[\beta(n+1 ; \xi)+(-1)^{n} \beta(n+1 ;-\xi)\right]
$$


or

$$
\beta(2 n+1 ; \xi)+\beta(2 n+1 ;-\xi)=\frac{(-1)^{n} e^{\pi i \xi}}{(2 n) !}\left(\frac{\pi}{2}\right)^{2 n+1} \mathcal{E}_{2 n}\left(e^{2 \pi i \xi}\right) .
$$

Further putting $\xi=0$ in (6.6), we arrive directly at the following well-known formula (see [1, p. 807, 23.2.22]):

$$
\beta(2 n+1)=\sum_{k=0}^{\infty} \frac{(-1)^{k}}{(2 k+1)^{2 n+1}}=\frac{(-1)^{n}}{2(2 n) !}\left(\frac{\pi}{2}\right)^{2 n+1} E_{2 n} .
$$

We can also obtain the formulas (6.2) and (6.7) by (4.11) and (4.15), respectively.

By Theorem 3.1. Theorem 3.2, Theorem 3.3 and Theorem 3.4, respectively, we easily find the following integral representations for the Apostol-Bernoulli and Apostol-Euler numbers:

$$
\begin{gathered}
\mathcal{B}_{n}\left(e^{2 \pi i \xi}\right)=-\Delta_{n}(0 ; \xi) \\
-n \int_{0}^{\infty} \frac{\cos \left(\frac{n \pi}{2}\right)\left(1-e^{-2 \pi t}\right) \cosh (2 \pi \xi t)+i \sin \left(\frac{n \pi}{2}\right)\left(1+e^{-2 \pi t}\right) \sinh (2 \pi \xi t)}{\cosh 2 \pi t-1} t^{n-1} \mathrm{~d} t
\end{gathered}
$$

$$
\begin{aligned}
& \mathcal{B}_{n}\left(e^{2 \pi i \xi}\right)=-\Delta_{n}(0 ; \xi)+\frac{2 n}{(-2 \pi)^{n}} \\
& \quad \times \int_{0}^{1} \frac{\cos \left(\frac{n \pi}{2}\right)(1-t) \cosh (\xi \log t)-i \sin \left(\frac{n \pi}{2}\right)(1+t) \sinh (\xi \log t)}{t^{2}-2 t+1}(\log t)^{n-1} \mathrm{~d} t,
\end{aligned}
$$

$$
\begin{aligned}
\mathcal{E}_{n}\left(e^{2 \pi i \xi}\right) & =2^{n+2} e^{-\pi i \xi} \\
& \times \int_{0}^{\infty} \frac{\cos \left(\frac{n \pi}{2}\right) \cosh \pi t \cosh (2 \pi \xi t)+i \sin \left(\frac{n \pi}{2}\right) \cosh \pi t \sinh (2 \pi \xi t)}{\cosh 2 \pi t+1} t^{n} \mathrm{~d} t
\end{aligned}
$$

$$
\begin{aligned}
\mathcal{E}_{n}\left(e^{2 \pi i \xi}\right) & =(-1)^{n} \frac{2^{n+2} e^{-\pi i \xi}}{\pi^{n+1}} \\
& \times \int_{0}^{1} \frac{\cos \left(\frac{n \pi}{2}\right) \cosh (2 \xi \log t)-i \sin \left(\frac{n \pi}{2}\right) \sinh (2 \xi \log t)}{t^{2}+1}(\log t)^{n} \mathrm{~d} t .
\end{aligned}
$$

Further setting $\xi=0$ in (6.8)-6.11), respectively, we deduce the integral representations for the classical Bernoulli numbers and Euler numbers as follows (see, e.g., [18, pp. 28-32]):

$$
\begin{aligned}
B_{n} & =-n \cos \left(\frac{n \pi}{2}\right) \int_{0}^{\infty} t^{n-1} e^{-\pi t} \operatorname{csch}(\pi t) \mathrm{d} t \\
& =\cos \left(\frac{3 n \pi}{2}\right) \frac{2 n}{(2 \pi)^{n}} \int_{0}^{1} \frac{(\log t)^{n-1}}{1-t} \mathrm{~d} t, \\
E_{n} & =2^{n+1} \cos \left(\frac{n \pi}{2}\right) \int_{0}^{\infty} t^{n} \operatorname{sech}(\pi t) \mathrm{d} t \\
& =\cos \left(\frac{3 n \pi}{2}\right) \frac{2^{n+2}}{\pi^{n+1}} \int_{0}^{1} \frac{(\log t)^{n}}{1+t^{2}} \mathrm{~d} t .
\end{aligned}
$$


Recently, Garg et al. 9] gave an extension of Apostol's formula (4.7) as

$$
\mathcal{B}_{n}(a ; \lambda)=-n \Phi(\lambda, 1-n, a) \quad\left(n \in \mathbb{N}, \lambda \in \mathbb{C},|\lambda| \leq 1, a \in \mathbb{C} \backslash \mathbb{Z}_{0}^{-}\right) .
$$

By (1.4) and the binomial theorem, we have

$$
\begin{aligned}
\sum_{n=0}^{\infty} \mathcal{E}_{n}(a ; \lambda) \frac{z^{n}}{n !} & =\frac{2 e^{a z}}{\lambda e^{z}+1}=2 \sum_{k=0}^{\infty}(-\lambda)^{k} e^{(k+a) z} \\
& =\sum_{n=0}^{\infty}\left[2 \sum_{k=0}^{\infty}(-\lambda)^{k}(k+a)^{n}\right] \frac{z^{n}}{n !} \\
& =\sum_{n=0}^{\infty}\left[2 \sum_{k=0}^{\infty} \frac{(-\lambda)^{k}}{(k+a)^{-n}}\right] \frac{z^{n}}{n !} .
\end{aligned}
$$

Hence, we also obtain the following interesting relationship between the ApostolEuler polynomials and the Hurwitz-Lerch zeta function:

$$
\mathcal{E}_{n}(a ; \lambda)=2 \Phi(-\lambda,-n, a) \quad\left(n \in \mathbb{N}, \lambda \in \mathbb{C},|\lambda| \leq 1, a \in \mathbb{C} \backslash \mathbb{Z}_{0}^{-}\right) .
$$

We can prove Theorem 2.1 and Theorem 2.2, respectively, by applying the relationships (6.12) and (6.14) in conjunction with Lerch's functional equation (4.8). The same as with the elementary series (4.12), we may also prove Theorem 4.1 and Theorem 4.2, respectively.

\section{REFERENCES}

1. M. Abramowitz, I. A. Stegun (Editors), Handbook of Mathematical Functions with Formulas, Graphs, and Mathematical Tables, National Bureau of Standards, Applied Mathematics Series 55, Fourth Printing, Washington, D.C., 1965. MR757537(85j:00005a)

2. T. M. Apostol, On the Lerch zeta function, Pacific J. Math. 1 (1951), 161-167. MR0043843 $(13: 328 \mathrm{~b})$

3. L. Carlitz, Multiplication formulas for products of Bernoulli and Euler polynomials, Pacific J. Math., 9 (1959), 661-666. MR0108601 (21:7317)

4. M. Cenkci, M. Can, Some results on $q$-analogue of the Lerch zeta function, Adv. Stud. Contemp. Math., 12 (2006), 213-223. MR2213080 (2007c:11098)

5. J. Choi, P. J. Anderson, H. M. Srivastava, Some $q$-extensions of the Apostol-Bernoulli and the Apostol-Euler polynomials of order $n$, and the multiple Hurwitz zeta function, Appl. Math. Comput. (2007), 199 (2008), 723-737. MR2420600

6. D. Cvijović, J. Klinowski, New formulae for the Bernoulli and Euler polynomials at rational arguments, Proc. Amer. Math. Soc., 123 (1995), 1527-1535. MR1283544(95g:11085)

7. D. Cvijović, The Haruki-Rassias and related integral representations of the Bernoulli and Euler polynomials, J. Math. Anal. Appl., 337 (2008), 169-173. MR2356063(2008i:33028)

8. A. Erdélyi, W. Magnus, F. Oberhettinger, F. G. Tricomi, Higher Transcendental Functions, Volumes I-III, McGraw-Hill, New York, 1953-1955. MR0698779 (84h:33001a) MR0698780 (84h:330016) MR0066496(16:586c)

9. M. Garg, K. Jain, H. M. Srivastava, Some relationships between the generalized ApostolBernoulli polynomials and Hurwitz-Lerch zeta functions, Integral Transforms Spec. Funct., 17 (2006), no. 11, 803-815. MR2263956 (2007h:11028)

10. H. Haruki, T. M. Rassias, New integral representations for Bernoulli and Euler polynomials, J. Math. Anal. Appl., 175 (1993), 81-90. MR1216746 (94e:39016)

11. D. H. Lehmer, A new approach to Bernoulli polynomials, American Math. Monthly, 95 (1988), 905-911. MR979133 (90c:11014)

12. R. Lipschitz, Untersuchung der Eigenschaften einer Gattung von unendlichen Reihen, J. Reine und Angew. Math. CV (1889), 127-156.

13. Q.-M. Luo, H. M. Srivastava, Some generalizations of the Apostol-Bernoulli and Apostol-Euler polynomials, J. Math. Anal. Appl., 308 (2005), 290-302. MR2142419 (2006e:33012) 
14. Q.-M. Luo, Apostol-Euler polynomials of higher order and Gaussian hypergeometric functions, Taiwanese J. Math. 10 (2006), 917-925. MR.2229631(2007c:33005)

15. Q.-M. Luo, H. M. Srivastava, Some relationships between the Apostol-Bernoulli and ApostolEuler polynomials, Comput. Math. Appl., 51 (2006), 631-642. MR.2207447 (2006k:42050)

16. Q.-M. Luo, An explicit relationship between the generalized Apostol-Bernoulli and ApostolEuler polynomials associated with $\lambda$-Stirling numbers of the second kind, Houston J. Math., accepted in press.

17. Q.-M. Luo, The multiplication formulas for the Apostol-Bernoulli and Apostol-Euler polynomials of higher order, Integral Transforms Spec. Funct., accepted in press.

18. W. Magnus, F. Oberhettinger, R. P. Soni, Formulas and Theorems for the Special Functions of Mathematical Physics, Third Enlarged Edition, Springer-Verlag, New York, 1966. MR0232968 (38:1291)

19. P. C. Pasles, W. A. Pribitkin, A generalization of the Lipschitz summation formula and some applications, Proc. Amer. Math. Soc., 129 (2001), 3177-3184. MR.1844990(2002e:11059)

20. H. M. Srivastava, Some formulas for the Bernoulli and Euler polynomials at rational arguments, Math. Proc. Cambridge Philos. Soc. 129 (2000), 77-84. MR1757780 (2001f:11033)

21. H. M. Srivastava, J. Choi, Series Associated with the Zeta and Related Functions, Kluwer Academic Publishers, Dordrecht, Boston and London, 2001. MR:1849375 (2003a:11107)

22. W. Wang, C. Jia, T. Wang, Some results on Apostol-Bernoulli and Apostol-Euler polynomials, Comput. Math. Appl., 55 (2008), 1322-1332. MR2394371

Department of Mathematics, East China Normal University, Shanghai 200241, People's Republic of China - And- Department of Mathematics, Jiaozuo University, Henan Jiaozuo 454003, People's Republic of China

E-mail address: luomath@126.com

E-mail address: luomath2007@163.com 